\title{
STUDY ON EXTRACTION METHODOLOGY OF RICE GROWTH CHARACTERISTICS IN FIELD USING TERRESTRIAL LASER MEASUREMENT DATA
}

\author{
K. Sakata ${ }^{1}$, A. Rikimaru ${ }^{1}$, Y. Yasunaga ${ }^{2}$, \\ ${ }^{1}$ Dept. Civil and Environmental Engineering, Nagaoka University of Technology, 1603-1 Kamitomioka, Nagaoka, Niigata, Japan - \\ sakata@vos.nagaokaut.ac.jp \\ ${ }^{2}$ Ecology Science co., ltd, 3-5-25 Kusoudzu Nagaoka, Niigata, Japan - yasunaga@zb.wakwak.com
}

Commission III, WG III/10

KEY WORDS: Terrestrial laser scanner, rice plant height, point clouds,DSM

\begin{abstract}
:
Smart agriculture is recommended for labor-saving, refinement and high quality production of rice cultivation with safety and security. The Terrestrial laser measurement has the advantage of acquiring 3D point cloud information with high accuracy and high density, but there is a disadvantage that it becomes difficult to measure the base of rice plant when paddy rice grew. In this study, the 3D point cloud information obtained by terrestrial laser measurement was used to three-dimensionally measure the growth state in the field corresponding to the growing period in time series. The growth characteristics of the whole field as a community were extracted as a representative value of mean growth. Then we attempted extraction of individual differences in growth and growth unevenness, as the deviation from the mean value of the field in terms of the growth characteristics of each strain focused on the individual plants. it is difficult to observe the root of the rice during the growing period, it was used for analyzing the growth quantity as the growth difference height for each growing period by obtaining the difference of the elevation value of the rice at the early stage of growth and at each stage of growing. The spatial variation in the growth quantity of the whole field area and the local area was calculated, the range of the deviation for evaluating the mean variation and the growth abnormality was examined, and the setting of the threshold for evaluating the growth normal and abnormality was examined.
\end{abstract}

\section{INTRODUCTION}

In Japan, smart agriculture aiming at maximum production yield, production control using ICT technology advanced was recommended. Remote sensing technology has attracted attention for precise cultivation related to smart agriculture. For example, Inoue and Yokoyama (2017) has proposed a field crop and farmland diagnosis using a drone equipped with an optical sensor. N. Tilly et al. (2014) suggests estimating biomass and height of corn using a terrestrial laser scanner. In this research, we are aiming to grasp the growth condition of paddy rice using Terrestrial Laser Scanner (TLS) which is excellent in portability and can acquire three dimensional information in a wide range. It suggested that the growth survey is possible using the surface height of paddy rice by TLS. The bottom end and the top end of paddy rice detected from the TLS viewed directly under and evaluated as rice plant height, indicating that its effectiveness is high. In this research, using this TLS, we examined the extraction method of rice growth characteristics considering the influence of measurement characteristics from oblique measurement. As growth characteristics, paddy rice has individual differences in growth, and it is useful to detect the difference in growth in its solid difference. Therefore, by using TLS that can be precisely measured, it is possible to extract growth characteristics using values related to plant height.

In presently, acquisition method of rice growth information was mean value that measured ten plant of plant height, leaf color, stem number in a field area.

Currently, as for the method of acquiring the growth information, 10 plants height, leaf colour, stem number are measured for one field, and they are averaged to represent the field value.
Examination of the total number of fields is difficult due to human factors, but it is useful as a simple representative value extraction. However, if there are individual differences even in the 10 stocks to investigating, the representative values of the extracted rice stocks are dangerous in the whole field.

Therefore, we examined a method of extracting information on the growth characteristics of rice according to the index value without ground effect using TLS is excellent in portability and can measure area. In TLS measurement, there is no human measurement error, it has objectivity and reproducibility, and it is possible to conduct a survey of all stocks through a planar observation. Based on this planar measurement information, we attempted a method of extracting growth information taking individual differences of paddy rice into consideration.

\section{METHODS}

\subsection{Study area}

The study site is located at a paddy field of Nagaoka Niigata, Japan (N37²5'3', E138 47'50')).

In this study, 3D point clouds were acquired using RIEGL VZ 400. In order to obtain the point group in detail, the observation pitch angle was set to $0.02\left[^{\circ}\right]$. The TLS measurement is from the rice planting stage to the harvesting stage and is carried out on June 21, July 1, July 11, July 19, July 29, August 9, August 23, September 13 2016, it measured eight times in total. Manual measurement to measure plant height, plant stem, and leaf color on the same day.

In order to extract the growth characteristics, growth conditions were managed by fertilizer and grown. The classification of fertilizing quantity is shown in Fig.1. The A compartment applied normal fertilizer to the basic fertilizer, the B compartment set the 
basic fertilizer to half of usual, and there was no basic fertilizer in the C compartment. We applied fertilizer as usual in A1, B1, C1 compartment, half of fertilizer was applied in A2, B2, and fertilizer was not applied in $\mathrm{C} 2$ compartment. The A1 compartment is a standard fertilizer application. Rice plants are May 16th and additional fertilizer is July 22. Paddy field measurement by TLS was set 6 points, and 6 points data were composited. The standard deviation of the height of each observation day was about $2 \mathrm{~mm}$ with the horizontal board installed at the four corners of the field.

\begin{tabular}{|c|c|c|c|c|c|}
\hline \multirow{2}{*}{ Fertilization } & \multirow{2}{*}{$\begin{array}{l}\text { Base } \\
\text { Fertilizer } \\
{[\mathrm{kg}]}\end{array}$} & \multirow{2}{*}{$\begin{array}{l}\text { Additional } \\
\text { Fertilizer } \\
{[\mathrm{kg}]}\end{array}$} & $\mathbb{D}$ & $\mathbb{D I}$ & (II) \\
\hline & & & \multirow{5}{*}{ C1 } & \multirow{5}{*}{ B1 } & \multirow{5}{*}{ A1 } \\
\hline $\mathrm{A} 1$ & 3.00 & 1.92 & & & \\
\hline $\mathrm{B} 1$ & 1.50 & 1.92 & & & \\
\hline $\mathrm{C} 1$ & 0.00 & 1.00 & & & \\
\hline $\mathrm{A} 2$ & 3.00 & 1.00 & & & \\
\hline B2 & 1.50 & $1.00 \quad \uparrow$ & & & \\
\hline $\mathrm{C} 2$ & 0.00 & 0.00 & & & \\
\hline \multicolumn{3}{|c|}{$\begin{array}{l}\text { The Quantity of Nitrogen } \\
\text { per } 1000 \mathrm{~m}^{2}\end{array}$} & $\begin{array}{c}\mathrm{C} 2 \\
10 \mathrm{~m}\end{array}$ & B2 & $\mathrm{A} 2$ \\
\hline
\end{tabular}

Fig.1 Experimental site and fertilization blocks

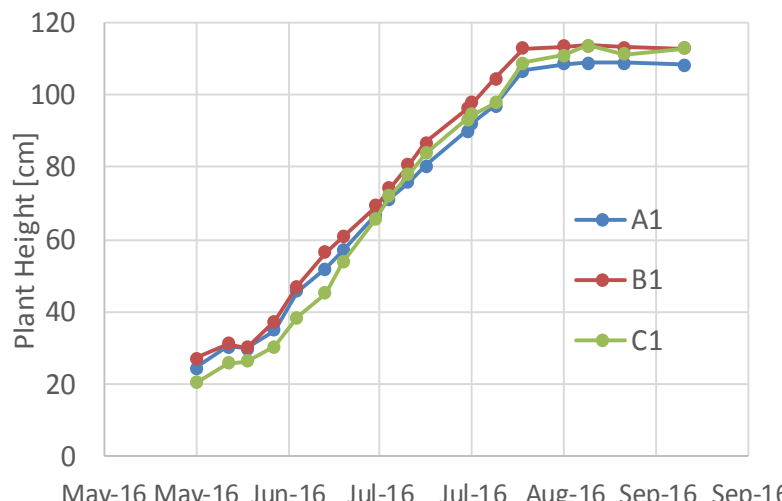

Fig. 2 manually measured rice plant height

\subsection{Generating DSMs}

TLS measurements were taken at six points from the field fairway, and the six data were combined.

For the conversion to the DSM raster image, the rice point group data acquired by TLS was taken as the $99 \%$ tile value in the $5 \mathrm{~cm}$ mesh. In order to remove noise components above the paddy field, the tile value was $99 \%$.
DSM : 99 percentile height each local area Local area size is $50 \mathrm{~mm}$

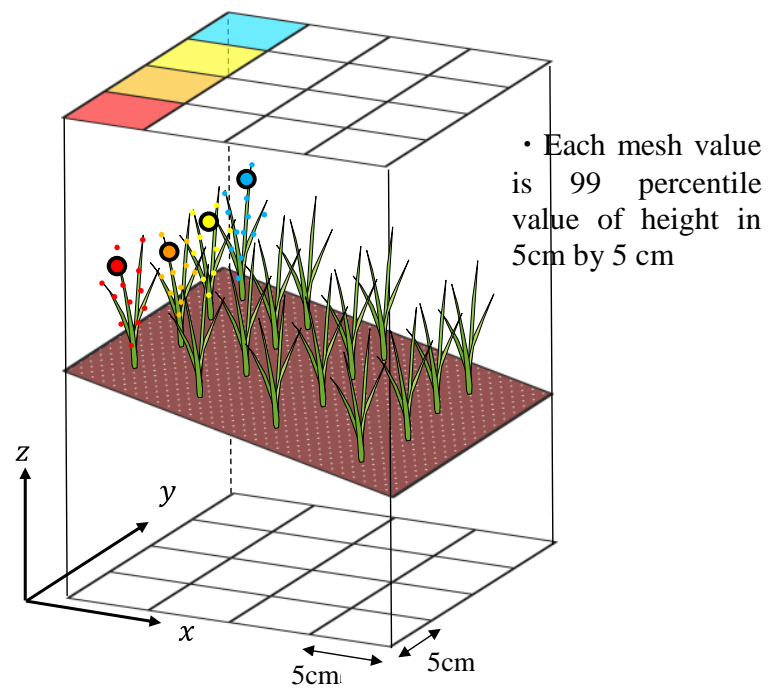

Fig.3 Experimental site and fertilization blocks

\section{RESULT}

Extraction method of rice growth characteristics using 3D point cloud from TLS measurement is below.

- Calculating rice different plant height

- Extraction rice growth characteristics

\subsection{Calculation of rice different plant height}

In the oblique measurement from TLS used in this research. The laser is obliquely irradiated to paddy rice, when it grows, due to the shielding of the ground by the leaf surface, the influence of the inclination and roughness of the ground, the practice with measurement; it is difficult to extract the height from the ground from the three-dimensional point cloud information of TLS.

Phan et al (2016) have constructed a regression model with plant height using the relative vertical distance of rice with laser measurement data in direct viewing. In the calculation of the relative vertical distance, when the shielding effect by the leaf surface is high at time of early growth period, the lower layer became unstable. Therefore, as shown in the Fig 4, the height difference value was calculated with respect to the change in the surface layer height of the rice bowl with the measurement height at the initial stage of growth as the reference height. By using this method, it is also possible to calculate the growth quantity without considering the inclination and irregularities of the ground. In this study, we analyzed the rice differential difference $\mathrm{RDH}$ as the growth quantity for each observation day. The formula for calculating RDH is shown in Equation 1. 


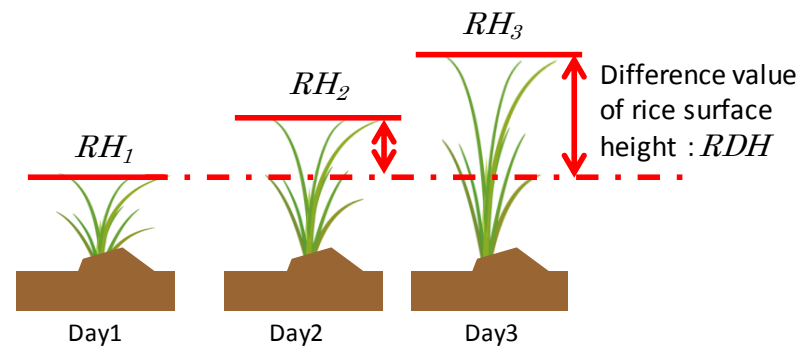

Fig.4 Image of RDH

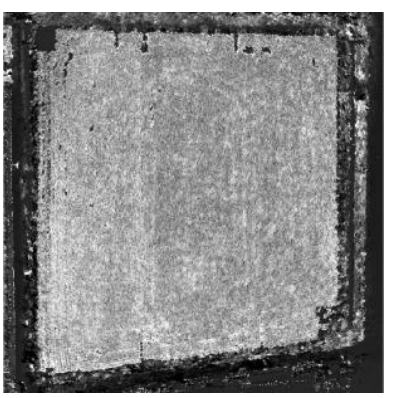

Jul-1

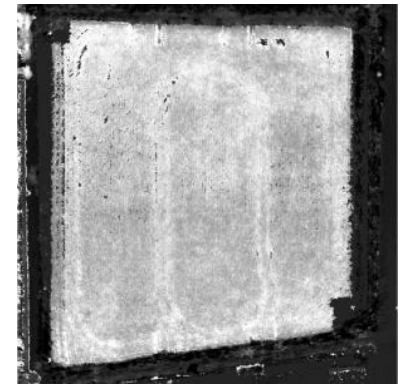

Aug-9

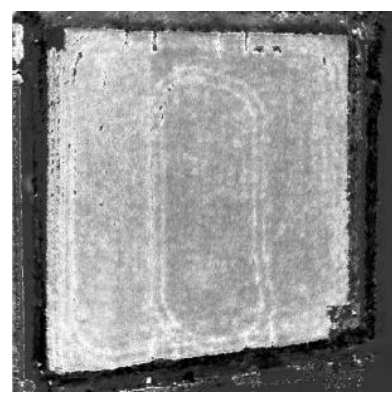

Jul-11

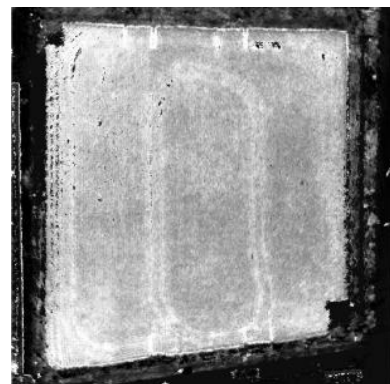

Aug-23

$$
\begin{aligned}
R D H & =R H_{i}-R H_{1} \quad(1) \\
\text { where } R D & =\text { Rice Different Height }[\mathrm{m}] \\
R H & =\text { Rice Height on local area }[\mathrm{m}] \\
i & =\text { measurement day }
\end{aligned}
$$

The image of RDH calculated based on the first observation date is shown in Fig 5.

The image on September 13 has areas where rice is lodged and RDH is calculated low.

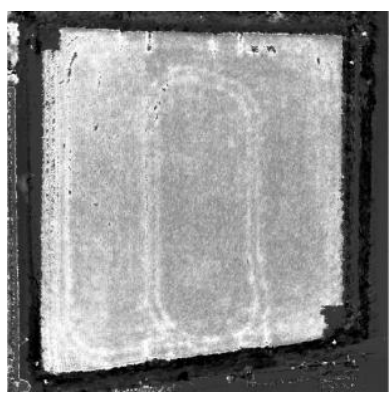

Jul-19

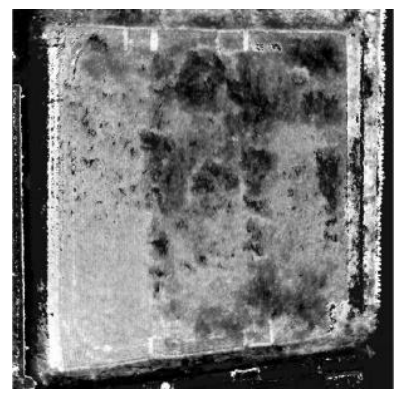

Sep-13

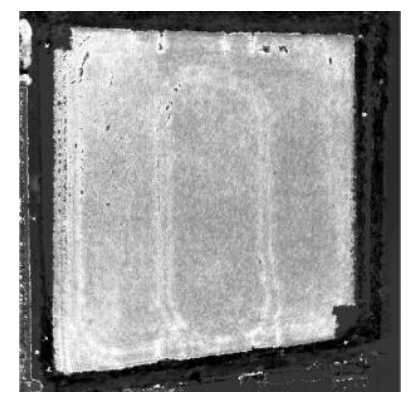

Jul-29

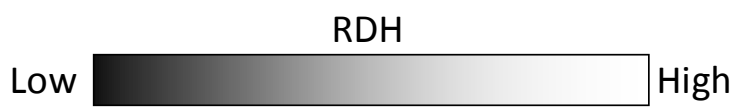

Fig.5 Images of RDH

For verification of RDH, it was checked against PDH calculated from practice measurement. For the calculation of the difference value, it is necessary to detect the local highest surface layer height $\mathrm{RH}$ of the rice plant. However, when growing, the position of $\mathrm{RH}$ changes within the range of one share depending on the curvature of the foliage and the way in which the rice plant collapses. Therefore, in order to cope with the change in the travel distance of RH, we developed a matching method in this research. Fig. 6 shows the method of searching for the highest surface layer in the matching method.

As shown in Fig. 6, the initial place of practice measurement was set, and the highest value within the search window centered on that place was extracted. The maximum value extracted was calculated as the surface layer height at the observation day as the difference value of the surface layer height from the beginning. In this study, it is assumed that the search window falls within the search window that is compatible with the inter-strain and inter-section, and the maximum height is the plant height with one stock. In the target field, the distance between plants is about $20 \mathrm{~cm}$ and the distance between strands is about $30 \mathrm{~cm}$, so the

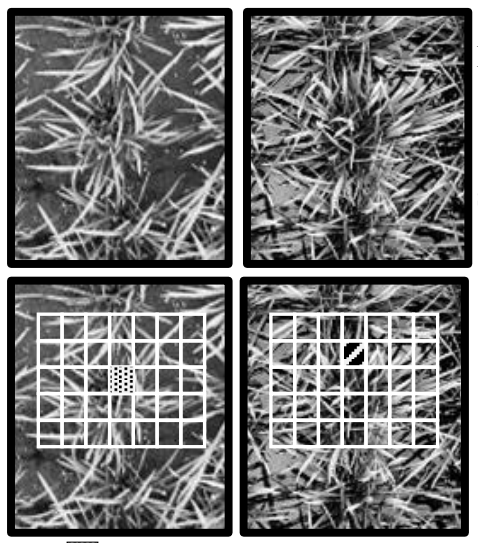

节 First search position

Maximum DSM position of each observation day
Maximum DSM position was changing in each observation day. DSM position was searched by local windows

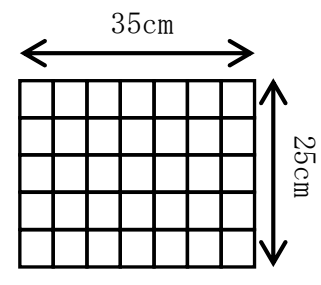

Search window
Fig. 6 Plant height search method 
search range is set to $25 \mathrm{~cm} \times 35 \mathrm{~cm}$. For practical measurement, the value of plant height was used to obtain the plant height difference value from the beginning. The differences between the plant height difference value and the manual measurement plant height were checked each other. The manual measurement plant different height was calculated by Equation 2. Fig.7 shows the RDH and PDH excluding September 13, which is calculated by this method and is the ripening period. The reason for excluding the ripening stage is to measure the height of the crown when the rice plant is lodged when it is ripened, whereas in practice measurement the rice cultivation stands and the difference is shown.

$$
\begin{aligned}
& P D H=P H_{i}-P H_{1} \quad \text { (2) } \\
& \text { where } P D H=\text { Plant height different height } \quad[\mathrm{m}] \\
& \mathrm{PH}=\text { manual measurement plant height }[\mathrm{m}] \\
& i=\text { measurement day }
\end{aligned}
$$

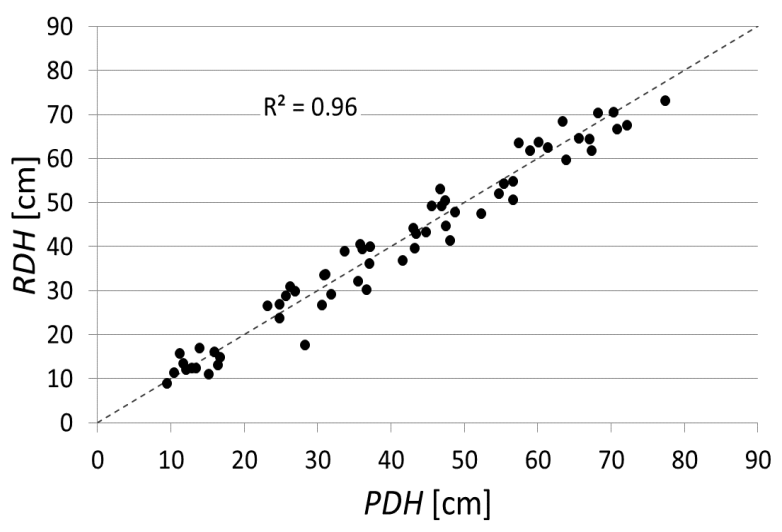

Fig.7 Compare RDH and PDH

The coefficient of determination between RDH and PDH was 0.96. The RMSE of PDH and RDH was $3.5 \mathrm{~cm}$.

\subsection{Extraction rice growth characteristics}

The variation in growth of each fertilizing area is defined as Rice growth unevenness. In this paper, fertilization conditions are different for each area, and due to the difference in growing situation, we compare the variation of the growing state.

The growth situation is judged by measuring the individual stock. However, depending on the measurement place of the individual stock, there is a danger to the representative value of the field because there are individual differences. Therefore, by using TLS we examined the extraction method of the growth characteristics using the variation about the difference of the growth. As shown in the Fig.8, when rice plants are delayed in growth due to individual differences and there are small rice stocks or rice stocks growing quickly and growing large, on the contrary from the average value of the standard growing plant height, the plant height becomes small or large. We propose a method to judge the growth condition of the plant height by using the standard deviation showing the variation. The histogram for each fertilizing area is shown in Fig.9. It understands that the value of $\mathrm{RDH}$ increases with each growth in each section. The threshold value is set by using $\mu$ and $\sigma$. Differences in growth are judged from the histogram of fertilization conditions of each section and used as a threshold.

It was assumed that difference in growth due to individual differences appear spatially randomly, and growth unevenness

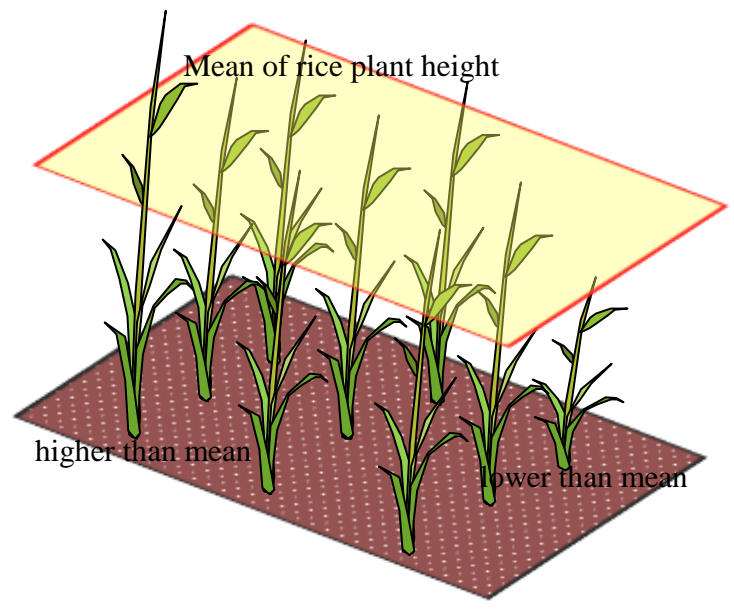

Fig. 8 Image of rice growth unevenness in a rice field

due to soil condition and fertilization conditions appears spatial clusters.

\subsection{Distribution images of growth characteristics}

Distribution images of growth characteristics was prepared using average value $\mu$ and standard deviation $\sigma$. Fig.10 shows the variation of growth using standard deviation. The average growth is gray color.

From Fig.10, small plant height regions in fertilization area are distributed in spatially cluster, so growth unevenness could be extracted. On July 1, the low growth area and the high growth area are sparsely distributed, but after July 11, the high area and the low area are solidified and distributed. It suggests that the growth difference gradually appears after July 11 .

The histogram of the fertilization area is shown in Fig.11. Regarding threshold setting Fig.11 A1 shows the histogram of A1 which is a standard habitat environment and $\mathrm{C} 2$ which does not give any fertilizer. The mode by RDH was almost the same in $\mathrm{A} 1$ and $\mathrm{C} 1$ section. In addition, separation was difficult even with the value of $\mu \pm \sigma$.

\section{DISCUSSION}

In this paper, we investigated the feature extraction method of growing condition due to the planar variation of field using TLS. In shown histogram of each fertilization area, since there was not much difference in standard deviation in each fertilization area, there was no change in growth unevenness. Fig. 12 shows time series of RDH. In the $\mathrm{C} 1$ and $\mathrm{C} 2$ sections without the base fertilizer, the slope of the community RDH during the busy period is large with respect to the A section and the B section. However, from the practice measurement, the height of the plant is larger in the A section and B section. In this study, the RDH is calculated as the difference value from the initial plant height, but if the growth until this initial observation day is large, the RDH after that tends to decrease. In this study, it was caused by the fact that it was growing at the early observation days in the A and $\mathrm{B}$ plots. On the other hand, because there was no base fertilizer in the $\mathrm{C} 1$ and $\mathrm{C} 2$ area, there was a delay in growth at the initial stage, and RDH was calculated largely because it grew after the initial measurement. We attempted to extract growth characteristics using $\mu \pm \sigma$, but from the histogram of Fig. 12, it became difficult to detect a clear threshold value due to the influence of initial observation. However, it was suggested that by using $\mu \pm \sigma$, it is possible to grasp the distribution of growth variation in each 
section. In addition, according to the distribution map, it is possible to understand areas where growth delay and growth are earlier than average growth. It is understood that the region where growth is gradually increasing is gradually increasing.

\section{CONCLUSION}

We investigated a method of extracting variation of growth in a field with different fertilization conditions by using RDH index without influence of ground height. It was suggested that by using $\mu \pm \sigma$, it is possible to grasp distribution such as growth variation in each section. Growth unevenness was extracted in spatially cluster area. For the RDH, it is found that the timing of the initial observation becomes important. In the histogram of A1 and $\mathrm{C} 2$, too much difference was not confirmed in the variation. Growth diagnosis with only plant height is dangerous, and comprehensive judgment is required such as leaf color and number of stems.

It was confirmed that the variance was small in the whole section by conducting measurement survey of all plant height using laser.

\section{REFERENCE}

Yoshio Inoue, Masaki Yokoyama, Drone-based Remote Sensing of Crops and Soils and its Application to Smart Agriculture, Journal of the Remote Sensing Society of Japan, Vol.37, No.3,224-235(2017)

N. Tilly et al, Evaluation of terrestrial laser scanning for rice growth monitoring, Inc. Arch. Photogramm. Remote Sens. Spat . Inf. Sci. XXXIX-B7 , 351-356 (2012)

N. Tilly et al ,Terrestrial Laser Scanning For Plant Height Mesurement and Biomass Estimation of Maize , Inc. Arch. Photogramm. Remote Sens. Spat . Inf. Sci. VII-7 , 181-187 (2014)

Anh Thu Thi Phan et al, Method for estimating rice plant height without ground surface detection using laser scanner measurement, J.Appl.Remote Sens., 10(4), 046018(2016), doi:10.117/1.JRS.10.046018
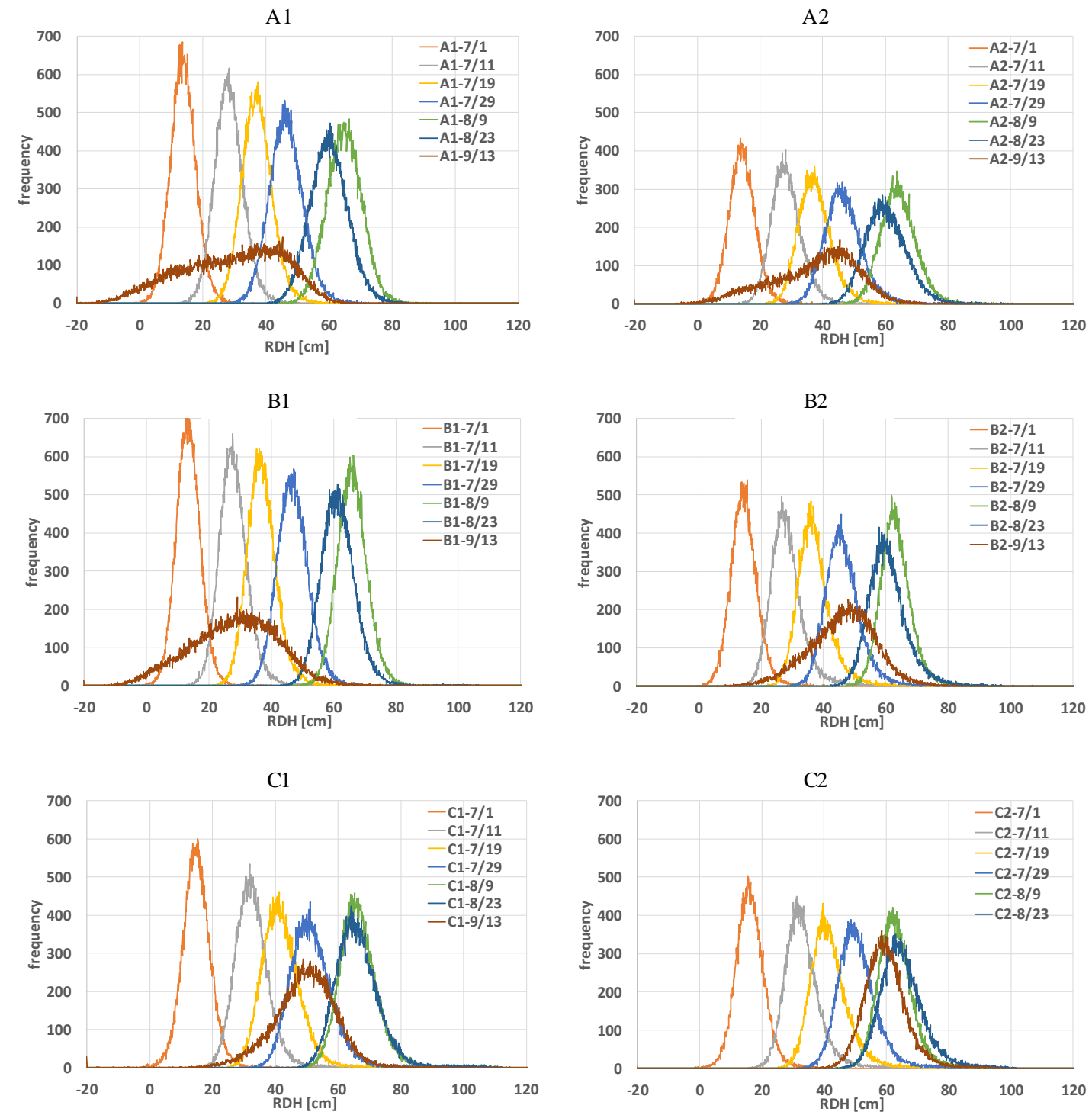

Fig.9 Histogram of each fertilization blocks 


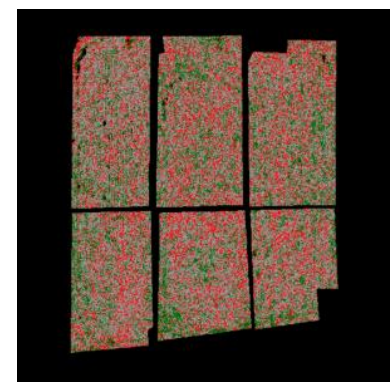

Jul-1

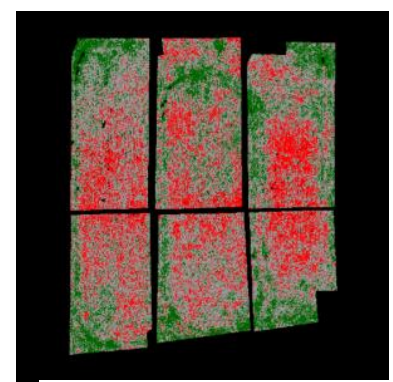

Aug-9

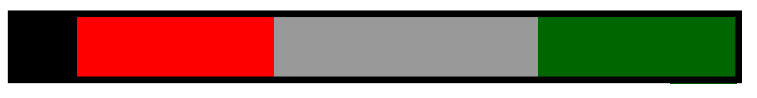

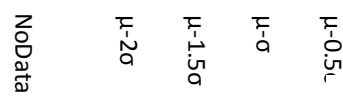

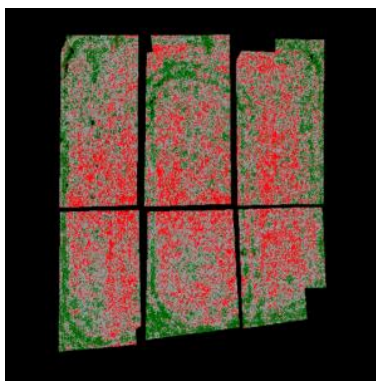

Jul-11

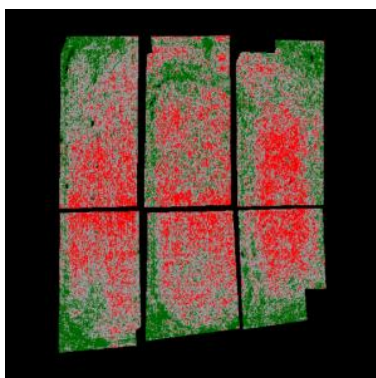

Aug-23

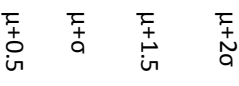

Fig.10 Images of rice growth unevenness

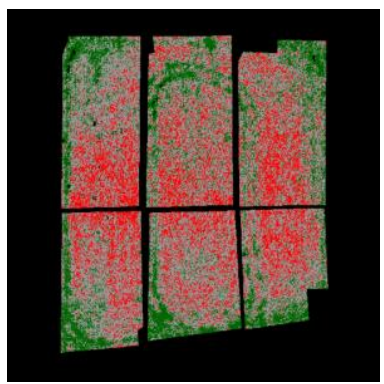

Jul-19

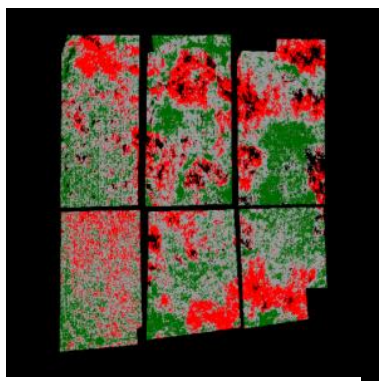

Sep-13

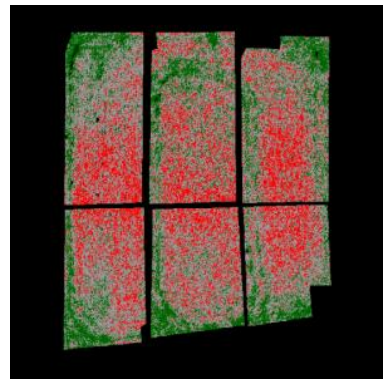

Jul-29

Set the threshold with $\pm 1 \sigma$
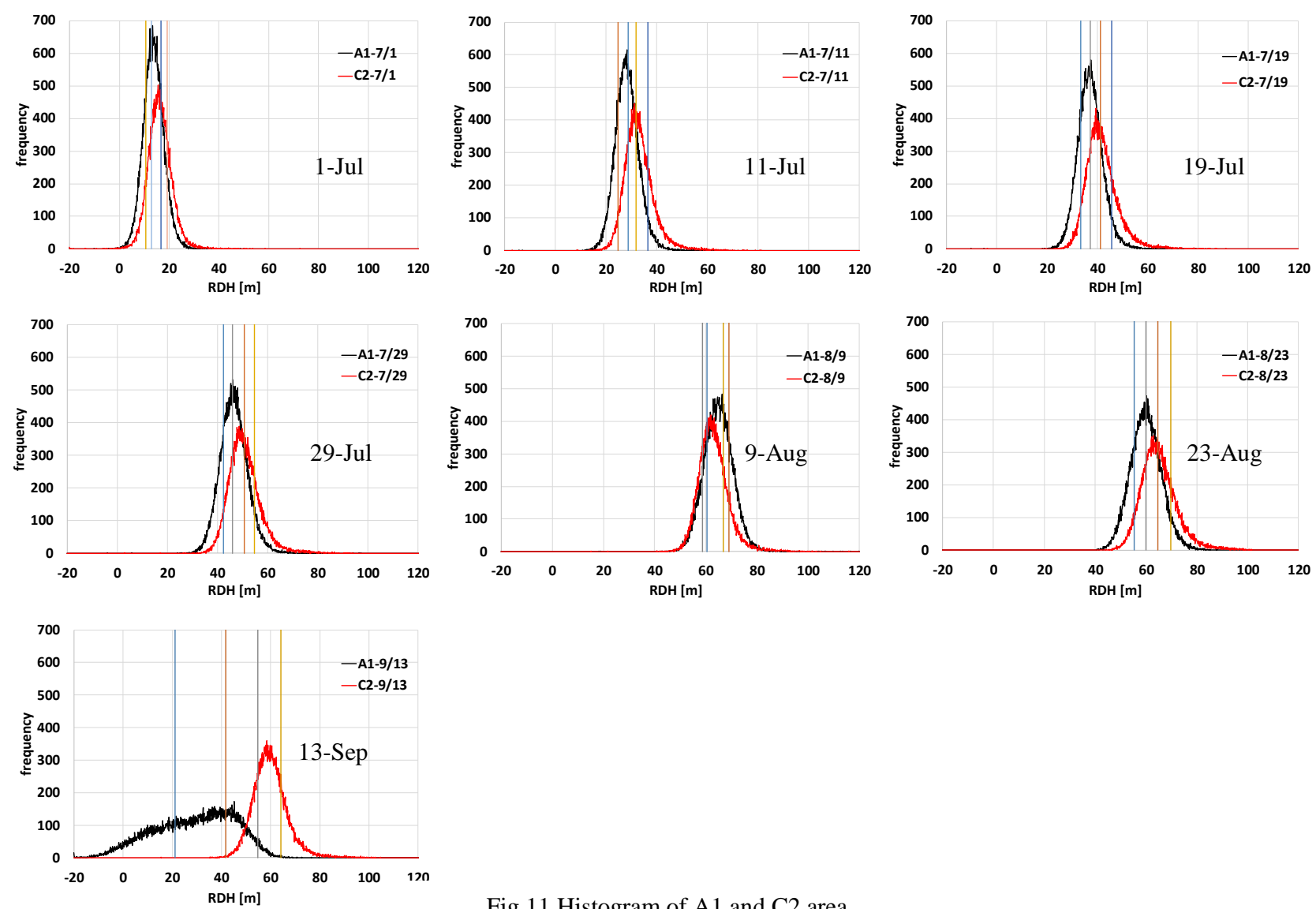

Fig.11 Histogram of A1 and C2 area 


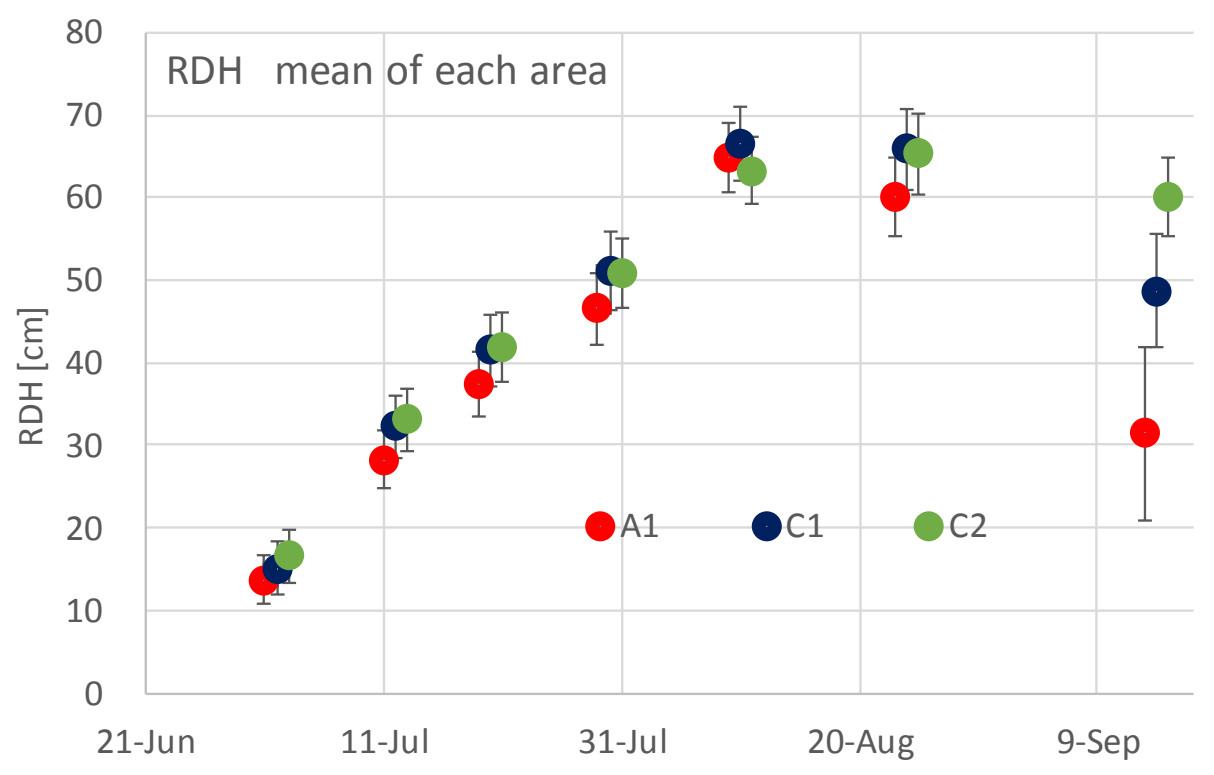

Fig.12 Time-series changing of RDH 